Revue d'histoire de l'Amérique française

DE REVUE D.HISTOIRE DE L'AMÉRIQUE FRANÇAISE

\title{
Introduction. Le passé des autres : lectures, emprunts et appropriations en contexte québécois
}

\section{Daniel Poitras et Maxime Raymond-Dufour}

Volume 71, numéro 3-4, hiver-printemps 2018

Le passé des autres : lectures, emprunts et appropriations en contexte québécois

URI : https://id.erudit.org/iderudit/1048510ar

DOI : https://doi.org/10.7202/1048510ar

Aller au sommaire du numéro

Éditeur(s)

Institut d'histoire de l'Amérique française

ISSN

0035-2357 (imprimé)

1492-1383 (numérique)

Découvrir la revue

Citer ce document

Poitras, D. \& Raymond-Dufour, M. (2018). Introduction. Le passé des autres : lectures, emprunts et appropriations en contexte québécois. Revue d'histoire de l'Amérique française, 71(3-4), 5-11. https://doi.org/10.7202/1048510ar d'utilisation que vous pouvez consulter en ligne. 


\title{
Introduction
}

\section{Le passé des autres : lectures, emprunts et appropriations en contexte québécois}

DANiEL Poitras

Université de Toronto

Maxime Raymond-Dufour

Université du Québec à Trois-Rivières

\begin{abstract}
S'intéresser au passé des autres implique davantage que la recherche $\checkmark$ de dépaysement dans un folklore plus ou moins exotique. La façon d'envisager ce passé et les représentations, emprunts et appropriations qui en découlent sont au cœur de ce numéro de la Revue d'histoire de l'Amérique française. Dans les articles qui suivent, ces «autres" sont les révolutionnaires belges, la droite catholique mexicaine, l'institution de la psychiatrie en France et aux États-Unis, les promoteurs de l'anglais comme langue universelle, les Franco-Américains et le maoïsme en Chine. Les auteurs de ce numéro thématique nous montrent comment les acteurs québécois reprennent à leur compte l'histoire d'ailleurs pour donner du sens au passé et au présent $d^{\prime}$ «ici» ${ }^{1}$. Plus largement, ces six contributions
\end{abstract}

1. À titre indicatif, voici quelques autres exemples de ces lecteurs : les missionnaires (Éric Desautels, "La représentation sociale de l'Afrique dans le discours missionnaire canadien-français (1900-1968)", Mens, 13, 1 (2012), p. 81-107; et Catherine Legrand, «L'axe missionnaire catholique entre le Québec et l'Amérique latine. Une exploration préliminaire», Globe, 12, 1 (2009), p. 43-66); les revues culturelles: Michel Lacroix, «La francophonie en revue, de La Nouvelle Relève à Liberté (1941-1965)», Globe, 14, 2 (2011), p. 37-58; les écrivains: voir le numéro de Globe, "Américanités francophones. Ancrages médiatiques, mises en perspective historiques et comparatistes », 7, 2 (2004); les chercheurs: François-Olivier Dorais, "Présence et influence de Robert Mandrou au Québec», Revue d'histoire de l'Amérique française, 69, 3 (2016), p. 59-82; les musiciens : voir la thèse (en préparation) d’Eric Fillion, «Listening for Resonance and Consonance: Music in Canadian-Brazilian Relations (1940s-1960s)", Université Concordia, histoire; et les organisations et mouvements sociaux: Karen Dubinsky, Babies without Borders : Adoption and Migration 
nous invitent à réfléchir sur la place faite au passé des autres à la fois dans l'historiographie et au Québec en général.

Le particularisme attribué au Canada français et, par la suite, au Québec, a joué un rôle clé dans le déploiement, d’une décennie à l'autre, des lectures du passé des autres. François-Xavier Garneau, par exemple, souhaitait

que les Canadiens soient fidèles à eux-mêmes; qu'ils soient sages et persévérans, qu' ils ne se laissent point emporter par le brillant des nouveautés sociales ou politiques. Ils ne sont pas assez forts pour se donner carrière sur ce point. C'est aux grands peuples à essayer les nouvelles théories. Ils peuvent se donner des libertés dans leurs orbites assez spacieuses. Pour nous, une partie de notre force vient de nos traditions; ne nous en éloignons ou ne les changeons que graduellement ${ }^{2}$.

Cette citation est devenue célèbre parce que certains historiens y ont vu le coup d'envoi du paradigme de la survivance. Dans le Canada de l'Union, à la suite de l'échec des Rébellions, les Canadiens, francophones et catholiques, ne devaient-ils pas aménager l'avenir en s'appuyant sur le passé et en évitant de se lancer dans les grandes expériences de la modernité ? Or, la portée de cette citation ne se limite pas à sa seule conclusion: entre autres choses, elle livre une certaine compréhension de l'expérience historique qui, par la comparaison, amène à apprécier sa propre différence. En effet, si, selon Garneau, les Canadiens ne doivent pas se lancer dans de grandes expérimentations sociales et politiques, c'est parce que leur condition est différente de celle des "grands peuples" ${ }^{3}$. Cette com-

across the Americas (Toronto, University of Toronto Press, 2010); Rosanne Waters, "African Canadian Anti-Discrimination Activism and the Transnational Civil Rights Movement, 1945-1965", Journal of the Canadian Historical Association, 2 (2013), p. 386-424; et le numéro spécial du Bulletin d'histoire politique, "Les années 1960: quand le Québec s'ouvrait sur le monde», 23, 1 (2014). Voir également l’introduction de Maurice Demers pour le numéro spécial de Mens, «S’approprier le passé des autres: les usages de l'histoire internationale au Québec avant la Révolution tranquille», 13, 1 (2012), p. 7-18.

2. François-Xavier Garneau, Histoire du Canada depuis sa découverte jusqu'à nos jours, Tome quatrième (Québec, John Lowell, 1852), p. 317.

3. Pour sa part, Louis-Joseph Papineau semblait avoir trouvé une similarité particulièrement stimulante entre l'expérience irlandaise et le cas bas-canadien. Il y trouvait un cas similaire de "petite nation" dominée par la Grande-Bretagne (Yvan Lamonde, "Conscience coloniale et conscience internationale dans les écrits publics de Louis-Joseph Papineau (1815-1839)", Revue d'histoire de l'Amérique française, 51, 1 [1997], p. 17-21). À titre d'exemples de l'appropriation de l'histoire de ces "grands peuples», voir David McKeagan sur les tensions entre l'appropriation du passé des études économiques de la France et des États-Unis au Québec, «The First Fifty Years of the École des Hautes Études Commerciales de Montréal : from "School of Higher Studies" to University Business School», Historical Studies in Education, 1 (2014), p. 1-25, et Ivan Carel sur l'appropriation des ruptures symboliques françaises, «L'invention de la "Grande Noirceur” : la voie française », dans Xavier Gélinas et Lucia Ferretti, dir., Duplessis : son milieu et son époque (Québec, Septentrion, 2010), p. 36-52. 
préhension de l'histoire de Garneau est traversée d'une comparaison implicite entre peuples. L'histoire des autres est ici instrumentale dans l'élaboration de l'histoire de soi. L'expérience de l'altérité conditionne le jugement historique, tout comme l'élaboration d'une vision de l'avenir.

Rares sont les histoires qui se vivent et s'écrivent en autarcie, si même une telle chose existe. Pour Garneau, le soi est d'abord et avant tout national: il écrit l'histoire des Canadiens, ces habitants francophones de la vallée du Saint-Laurent, dont il cherche à orienter l'avenir collectif. Cela dit, les dimensions du soi sont mouvantes et variables, elles changent selon les personnes et selon les époques, elles se reconfigurent d'après les besoins culturels comme les désirs militants ${ }^{4}$. Peu importe leurs délimitations, elles nous confrontent nécessairement a contrario à ce qu'il y a au-delà de leurs limites comme à ce qui les a conditionnées.

À chaque délimitation ou assignation du "soi», de nouvelles limites se sont posées (explicitement ou non) et ont conditionné l'éloignement ou la proximité de ce qui relève de l'altérité. À cet égard, la lecture du passé des autres nous invite à une relecture critique de ce qui est considéré comme "propre " à une histoire. Le cas des révolutions de la fin du XVIII et de la première moitié du XIX ${ }^{e}$ siècle constitue un exemple éclairant: leur réagencement sous l'expression de "révolutions atlantiques ${ }^{5}$ » a en effet incité les chercheurs à décortiquer certains mythes, téléologies ou thèmes associés à l'État-Nation et réputés incomparables ${ }^{6}$. Cet étirement des frontières en vue de découpages neufs a favorisé l'étude de réseaux d'influences et de passeurs culturels au cour de la diffusion de modèles et d'expériences dont le métissage tendait à flouter l'identification du «soi» et de l'«autre». Puisqu'elle met en jeu directement le rapport au temps et à l'espace, l'étude des lectures du passé des autres invite à l'exploration de courants historiographiques tels que l'histoire transnationale ou connectée et l'histoire de la circulation des personnes, comme des

4. Anne-Marie Thiesse explique en détail comment l'identité nationale fut conçue en Europe, à la fin du XVIII ${ }^{\mathrm{e}}$ siècle et au début du XIX ${ }^{\mathrm{e}}$ siècle. Elle démontre que la recherche de particularismes nationaux est un phénomène transnational et synchronique. Il s'agit là d'un raisonnement qui s'applique bien au Bas-Canada et au Québec, malgré le fait que l'Amérique du Nord ne soit pas considérée dans l'étude de l'auteure (Anne-Marie Thiesse, La création des identités nationales [Paris, Seuil, 1999]).

5. Voir le numéro spécial de la Revue d'histoire de l'Amérique française, «La Nouvelle-France et l'Atlantique ", 64, 3-4 (hiver-printemps 2011) et Michel Ducharme, Le concept de liberté au Canada à l'époque des Révolutions atlantiques, 1776-1838 (Montréal et Kingston, Londres, Ithaca, McGill-Queen’s University Press, 2010).

6. Sur cette déconstruction, voir Marcel Detienne, Comparer l'incomparable (Paris, Seuil, 2009). 
idées ${ }^{7}$. La vieille énigme de la "synchronicité» des rythmes de développement de certains groupes et collectivités parfois éloignés - ou, à l'inverse, celle du "contemporain du non-contemporain» dont parlait Reinhart Koselleck $^{8}$ - y trouve un nouvel éclairage ${ }^{9}$.

Au cours des trois dernières décennies, les débats sur la normalité et l'exceptionnalité du Québec ont mis à l'ordre du jour la réflexion sur les lectures du passé des autres, tout en démontrant à quel point ces dernières étaient situées dans le temps. Dans Genèse des nations et cultures du Nouveau Monde, Gérard Bouchard comparait les récits fondateurs de différentes "sociétés neuves", dont le Québec, l'Australie et le Mexique. L’approche comparative qu'il mettait en ouvre lui permettait d'identifier ce qu'il nommait les «illusions de la singularité», qui portent l'historien à voir de l'exceptionnel là où il $\mathrm{y}$ a des phénomènes partagés par différentes sociétés. Mais Bouchard faisait également un peu plus : il fouillait dans le passé des autres à la recherche de leçons pour jauger l'évolution du Québec. En proposant l'hypothèse d'un dynamisme historique propre aux sociétés neuves, l'auteur identifiait également les moments et les choix par lesquels l'histoire québécoise aurait pu prendre une direction davantage en phase avec la "modernité» et le "progrès». En valorisant ainsi rétrospectivement le volontarisme historique, Bouchard cherchait également, à l'entrée du nouveau millénaire, à rallumer la flamme vacillante de l'expérience moderne au Québec. Il s'agissait de "réenchanter» l'horizon de la Révolution tranquille et ses thèmes clés (l'audace collective, la maîtrise de soi, la confiance en l'avenir ${ }^{10}$. Cette lecture relevait de ce que François Hartog a nommé le régime d'historicité moderne, au sein

7. On peut trouver un bon exemple de l'utilisation de l'étude de la circulation des idées pour comprendre la réappropriation de l'histoire des autres dans le livre d'Aurélio Ayala et François Le Jeune sur l'interprétation par la presse française de la révolution avortée des Patriotes (Aurélio Ayala et François Le Jeune, Les Rébellions canadiennes de 1837 et 1838 vues de Paris [Québec, Presses de l'Université Laval, 2011]). Sur les différentes approches d'histoire comparée, croisée et connectée à travers des exemples tirés de l'histoire du Québec, voir Aline Charles et Thomas Wien, «Le Québec entre histoire connectée et histoire transnationale», Globe, 14, 2 (2011), p.199-221.

8. Reinhart Koselleck, Le futur passé. Contribution à la sémantique des temps historiques (Paris, EHESS, 1990).

9. À cet égard, les travaux sur les similarités entre l'histoire du Québec et celles du Mexique, de l'Irlande ou de l'Australie, notamment, démontrent la pertinence de la thématique que nous proposons. Voir Maurice Demers, Connected Struggles. Catholics, Nationalists, and Transnational Relations between Mexico and Quebec, 1917-1945 (Montréal et Kingston, McGill-Queen’s University Press, 2014); Simon Jolivet, Le vert et le bleu : identité québécoise et identité irlandaise au tournant du XXe siècle (Montréal, Presses de l'Université de Montréal, 2011); Gérard Bouchard, Genèse des nations et cultures du Nouveau Monde (Montréal, Boréal, 2000). Voir également Serge Granger, Le lys et le lotus : les relations du Québec avec la Chine de 16501950 (Montréal, VLB éditeur, 2005).

10. G. Bouchard, Genèse des nations... 
duquel les rythmes et évolutions semblent aimantés à une histoire qui entraîne toutes les collectivités (occidentales) vers l'avant ${ }^{11}$.

On peut aujourd'hui mesurer le chemin parcouru depuis les chantiers ouverts par Genèse. La diversification des regards se vérifie notamment par la multiplication des approches pour lire l'histoire des autres et s'en inspirer. Les protagonistes et objets privilégiés ne se réduisent plus aux nations ou aux sociétés prises en bloc - ou encore à l'un des acteurs collectifs par excellence de la modernité, l'État-Nation. La fragmentation du corps national et l'importance prise, ici, par les études transnationales et, là, par les recherches sur le postcolonial, invitent à élargir le spectre de ceux qui abordent ou s'approprient le passé des autres. Ces «lecteurs» sont autant des individus, des organisations, des groupes que des institutions. Leurs lectures, parfois conflictuelles, peuvent en retour générer, localement, des débats nouveaux ou inciter à réévaluer et à actualiser le «soi» à partir duquel ces lecteurs parlent ${ }^{12}$.

Cette variété de lecteurs invite en retour à prendre en compte qui ou quoi est «lu», instrumentalisé, ou approprié. Avec l'amenuisement des liens du national, les jeux de miroir se sont complexifiés, notamment grâce à la prise en compte du fait que l'«autre» a aussi ses rapports d'altérité. Loin de se fondre dans un récit national unique, ces autres de l'autre tiennent eux aussi, à partir du centre ou de la périphérie, des discours complexes sur leur «soi» tout en entretenant une mémoire particulière. Lorsqu'elle s'étale sur une période suffisamment longue, cette perspective démontre que la lecture de l'histoire des autres, tout en étant conditionnée par des postulats et des préjugés souvent tenaces, n’est ni figée ni déterminée. Sean Mills illustrait récemment, en étudiant la représentation des Haïtiens construite par les missionnaires pendant l'entre-deux-guerres et par les intellectuels des années 1960-1970, à quel point la lecture de l'histoire de l'autre (ici des Haïtiens réfugiés au Québec après avoir fui le régime Duvalier) se transforme en fonction à la fois d'un contexte provincial en pleine évolution et des stratégies de ces «autres» afin de mobiliser leur passé et d'en orienter la lecture par les Québécois ${ }^{13}$.

L'importance prise par les études sur la mobilité et les transferts, notamment sous l'impulsion de l'histoire postcoloniale, incite ainsi à considérer

11. François Hartog, Régimes d'historicité. Présentisme et expériences du temps (Paris, Seuil, 2003).

12. L'histoire des relations internationales est elle-même de plus en plus sensible à cette diversité d'acteurs. Voir notamment David Meren, «De Versailles à Niamey. Le patrimoine constitutionnel canadobritannique du Québec et sa participation au sein de la Francophonie, 1968-1970 », Globe, 13, 1 (2010), p. $99-124$.

13. Sean Mills, Une place au soleil: Haïti, les Haïtiens et le Québec (Montréal, Mémoire d’Encrier, 2016). 
l'utilisation du passé des autres "de l'intérieur», c'est-à-dire ceux qui ne se fondent pas dans le groupe majoritaire national (les minorités ethnoculturelles, les Autochtones, les étudiants étrangers, les réfugiés, les passeurs et visiteurs, etc.). Puisque ces utilisations impliquent différents rapports de force sur les plans social, culturel et politique, elles sont de bons indicateurs de l'agentivité historique qui est reconnue (ou non) à ces autres de l'intérieur $^{14}$. Leur passé est-il reconnu ou disqualifié, mis en dehors de l'histoire ${ }^{15}$ ? Sont-ils considérés comme possesseurs d'une mémoire porteuse et pertinente? Quelle place est accordée à leur passé dans les enjeux touchant à la société québécoise, notamment sur le plan de l'enseignement de l'histoire? Par quel processus un passé considéré comme "autre» devient-il un passé apprivoisé et parfois métissé avec le(s) passé(s) des groupes sur place, majoritaires ou non? Pour aborder ces questions, l'histoire orale a récemment mis en évidence une polyphonie de voix et de mémoires tenant à la fois à l'ici et à l'ailleurs, ce qui incite à réfléchir au rôle des historiens par rapport à différentes communautés de mémoire ${ }^{16}$.

On voit que les enjeux soulevés par les lectures du passé des autres invitent l'historien à faire preuve de réflexivité ${ }^{17}$. Appelé à questionner certaines de ses représentations de l'autre et, par là, du "soi» individuel ou collectif, l'historien est également poussé à réfléchir à la place qu’il occupe dans ce processus et au nom de quoi il procède à l'analyse, qu'il s'agisse de ses propres lectures du passé des autres ou de celles des lecteurs qu'il étudie.

Ce numéro thématique de la Revue d'histoire de l'Amérique française comporte six articles qui mettent en scène une diversité d'approches, de lecteurs et d' "autres». D’entrée de jeu, Maxime Raymond-Dufour étudie la lecture de l'histoire de la Révolution belge de 1830 faite par le politicien canadien Denis-Benjamin Viger. À travers les similarités qu'il perçoit entre cette révolution et le contexte bas-canadien, Viger relit l'histoire cana-

14. Brian Gettler a par exemple traité de l'instrumentalisation du passé des Autochtones afin de servir aux intérêts financiers de l'Empire ("En espèce ou en nature? Les présents, l’imprévoyance et l'évolution idéologique de la politique indienne pendant la première moitié du XIX siècle», Revue d'histoire de l'Amérique française, 65, 4 [printemps 2012], p. 409-437).

15. Les anthropologues ont particulièrement réfléchi à ces enjeux. Voir Johannes Fabian, Le temps et les autres. Comment l'anthropologue construit son objet (Toulouse, Anacharsis, 2006).

16. Voir Steven High et Catherine Foisy, «Un chantier à réinvestir ou à réinventer... Histoire contemporaine du Québec et sources orales ", Revue d'histoire de l'Amérique française, 69, 1-2 (été-automne 2015), p. 11-18.

17. À ce propos, voir les réflexions incontournables de Michael Werner et Bénédicte Zimmermann, "Penser l'histoire croisée: entre empirie et réflexivité», dans De la comparaison à l'histoire croisée (Paris, Seuil, 2004). 
dienne. Il l'agence différemment en s'inspirant de l'histoire belge afin de créer une nouvelle narration de l'histoire du Canada. Maurice Demers étudie de son côté la fascination de certains jésuites canadiens-français à l'égard de l'activisme catholique au Mexique. Dès le premier tiers du XX siècle, ce n'est pas du côté de l'Europe, mais vers le Sud que ces jésuites cherchaient, dans l'histoire mexicaine, des leçons afin de mettre en garde le public contre les dangers de la laïcisation et du communisme. Virginie Hébert s'intéresse, quant à elle, à la langue anglaise et au mythe voulant qu'elle soit la nouvelle lingua franca mondiale. L'auteure examine la réception de ce mythe chez divers lecteurs en fonction d'enjeux identitaires et éducatifs propres au Québec de l'entre-deux-guerres. Elle démontre que l'anglais, langue du "progrès ", permettait à plusieurs contemporains de questionner l'histoire et le devenir du Québec à travers la lecture d'un passé/mythe considéré comme extérieur.

Les trois derniers articles de ce numéro nous emmènent dans la seconde moitié du XXe siècle. Pierre Lavoie étudie l'interprétation des pérégrinations historiques des Franco-Américains par divers lecteurs dont l'industrie culturelle québécoise. Proches et lointains, les Franco-Américains sont devenus au cours du $\mathrm{XX}^{\mathrm{e}}$ siècle les vestiges incertains d'une époque révolue, ce qui amène l'auteur à examiner la dépossession identitaire de l'autre à travers une mise en scène qui verse dans l'anachronisme et le folklore. En retour, ce traitement permet à l'auteur de cerner l'évolution du rapport des Québécois francophones avec l'américanité et les ÉtatsUnis. Alexandre Klein, pour sa part, épie les voyages et les lectures de Camille Laurin, qui cherchait à réformer la psychiatrie au Québec en puisant dans les institutions française et américaine. L'auteur démontre comment l'histoire des autres a permis de complexifier la représentation de la maladie mentale et d'inspirer le projet d'une médecine nouvelle au Québec. Mais cette appropriation, loin d'être fixée, s'est également transformée au gré des réarrangements mémoriels effectués par Laurin. Enfin, Yuxi Liu s’intéresse au regard posé par des visiteurs québécois sur l'expérience communiste chinoise. Fantasmés selon les besoins locaux, ces "autres» ne servaient pas moins de truchements pour effectuer de nombreux séjours en Chine et pour organiser des initiatives maoïstes au Québec. Si l'ignorance de la Chine réelle jouait un rôle clé dans la lecture de son passé et de son présent, l'auteure démontre que la fascination pour cet autre lointain jouait dans les cordes d'un orientalisme qui touchait les Québécois bien au-delà des groupes d'extrême gauche. 